\title{
Power Allocation Optimization and Green Energy Cooperation Strategy for Cellular Networks with Hybrid Energy Supplies
}

\author{
Lin Wang ${ }^{1}$, Xing Zhang ${ }^{1,2}$ and Kun Yang ${ }^{1}$ \\ ${ }^{1}$ Wireless Signal Processing \& Networks Lab (WSPN), \\ Key Laboratory of Universal Wireless Communication, Ministry of Education \\ Beijing University of Posts \& Telecommunications (BUPT), \\ Beijing, 100876, China \\ ${ }^{2}$ Beijing Advanced Innovation Center for Future Internet Technology \\ Beijing University of Technology (BJUT), Beijing, China \\ [e-mail: zhangx@ieee.org] \\ *Corresponding author: Xing Zhang
}

Received March 23, 2016; revised June 23, 2016; accepted July 25, 2016; published September 30, 2016

\begin{abstract}
Energy harvesting is an increasingly attractive source of power for cellular networks, and can be a promising solution for green networks. In this paper, we consider a cellular network with power beacons powering multiple mobile terminals with microwave power transfer in energy beamforming. In this network, the power beacons are powered by grid and renewable energy jointly. We adopt a dual-level control architecture, in which controllers collect information for a core controller, and the core controller has a real-time global view of the network. By implementing the water filling optimized power allocation strategy, the core controller optimizes the energy allocation among mobile terminals within the same cluster. In the proposed green energy cooperation paradigm, power beacons dynamically share their renewable energy by locally injecting/drawing renewable energy into/from other power beacons via the core controller. Then, we propose a new water filling optimized green energy cooperation management-strategy, which jointly exploits water filling optimized power allocation strategy and green energy cooperation in cellular networks. Finally, we validate our works by simulations and show that the proposed water filling optimized green energy cooperation management strategy can achieve about $10 \%$ gains of MT's average rate and about $20 \%$ reduction of on-grid energy consumption.
\end{abstract}

Keywords: Energy harvesting, green energy cooperation, hybrid energy supplies, wireless energy transfer, water filling optimization

This work is supported by the National 973 Program under grant 2012CB316005, by the Fundamental Research Funds for the Central Universities 2014ZD03-01, by the National Science Foundation of China (NSFC) under grant 61372114, 61571054, 61471062, the New Star in Science and Technology of Beijing Municipal Science \& Technology Commission (Beijing Nova Program: Z151100000315077). 


\section{Introduction}

The continuous development of wireless communication technology combined with the deep optimization of the network has resulted in an exponential growth of mobile data traffic, which has contributed to a rapid increase in the energy consumption and carbon emission of the information and communication technology (ICT) sector. It is estimated that, in 2020, the annual carbon emission of the ICT industry will be 235 Mto [1] and the electric energy consumption will be 414 Twh [2]. This not only leads to enormous network operation cost, but also places heavy burdens on grid and the environment. Thus, reducing power consumption of the infrastructure within the system is crucial to cellular networks.

Multiple studies are providing strong support that the use of harvested renewable energy reduces the carbon footprint and on-grid power consumption of the cellular networks [3]-[5]. That because exploiting renewable energy to power facilities of cellular networks can reduce on-grid energy consumption. Energy harvesting (EH) from renewable energy sources (e.g., solar, wind, vibration, ambient radio power, etc.) is emerging as a practically appealing solution to prolong the lifetime of energy-constrained wireless networks [6], [7]. At the same time the renewable energy is more economical and green than conventional energy that generated from fossil fuels etc. [8], [9]. By introducing the harvested renewable energy to power the next-generation cellular networks, potentially $20 \%$ of $\mathrm{CO}_{2}$ emission can be reduced [10].

To enjoy the environmental friendliness and low-cost of the renewable energy, $\mathrm{EH}$ is an ideal solution. However, owing to the space-time instability and non-uniformity of green energy, it may not guarantee sufficient power supplies for facilities in the cellular networks. Thus, to overcome the unreliability of the renewable energy source, hybrid cellular networks powered by multiple types of energy supply (e.g., the on-grid energy, the solar energy, and the wind energy), where EH and the grid coexist, will be an ideal solution [4], [11]. In such cellular networks, the system is powered by renewable energy if the green energy collectors can gather enough power; otherwise, the facilities switch to on-grid energy.

With the development of energy harvesting circuit, power transfer in wireless communication systems has drawn significant attention. [12] propose a new network architecture for radio frequency charging stations, overlaying with an uplink cellular network. The paper described an energy transfer wireless communication system, in which energy can be delivered via microwave power transfer (MPT). The capability allows the wireless devices to harvest energy from microwave signals for information processing and transmission. We can use MPT to charge mobile terminals (MTs) and get rid of power cords. The study extended to a harvest-then-transmit architecture for power transfer in wireless broadcast system [13]-[15], and energy cooperation systems [16]-[25]. The works are studied in different system model and scenarios. In [13] the multi-antenna access point first broadcasts wireless power to all users via energy beamforming in the downlink, and then, the users send their independent information to the access point simultaneously in the uplink using their harvested energy. [14] lets the source and relay harvest energy from the access point in the downlink and work cooperatively in the uplink for the source's information transmission. [15] characterizes the spectral efficiency of an uplink radio frequency-powered macrocell network that the users transmit in the uplink while replenishing energy from base station in the downlink. [16] proposes an energy cooperation save-then-transmit scheme in a point-to-point wireless 
communications system, where energy is allowed to flow between the transmitter and receiver. A practical coordinated multipoint system with clustered base stations cooperatively communicating with mobile terminals is considered in [17] and [23]. [18] studies a model for energy cooperation between cellular base stations with hybrid power supplies, limited energy storages, and connected by resistive power lines for energy sharing. A cooperative mechanism for wireless energy harvesting and spectrum sharing in 5G networks is studied in [24], where secondary users harvest energy from both ambient signals and primary user's signals and can act as relays. [25] analyzes a cooperative energy efficiency model in cellular network under different cooperative transmission scenarios, interference levels and wireless channel conditions. Assuming the future channel side information is available, the maximum throughput of point-to-point EH fading channels can be achieved by the directional water filling algorithm [26]. For wireless networks with hybrid energy sources, a power allocation algorithm was proposed in [27] to achieve energy cost minimization. [28] shows us an optimal power allocation policy to minimize the conventional energy consumption with delay-constrained data traffic requirement in heterogeneous cellular networks. Transmission protocols for cellular networks with hybrid power supplies have also been studied in [4], [9], [11], [28], [29]. However, as the harvested green energy is snatchy and sporadic, the design of the network setting is challenging. Energy management decision in EH networks should be based on both the channel side and the energy side adaptively. Thus more decisions must be made, and more information will be needed.

In the prior works, the network's parameters can't be changed dynamically and a suitable strategy can't be chosen according to the variation of the channel state information (CSI) of the system. Moreover, in [12] all power beacons (PBs) have to deliver energy to MTs with the same transmission power, no matter how far the distance between PB and MT is. Much energy has been wasted, and due to the high energy transfer the addictive white noise is increased, both of them do harm to the performance of the system. To achieve agile response to the changes of the CSI, and overcome the shortage of non-adjustable transmission power of PB, in this paper, we introduce a dual-level control architecture to detect and manage the system dynamically. In the architecture, controllers are responsible for gathering information for a core controller (CC), and the CC grasps the entire system. After processing the collected data, the CC can adjust the parameters of the network according to the conditions of the system and send commands to PBs dynamically. We propose a power allocation optimization approach for cellular networks with hybrid energy supplies as shown in Fig. 1, where stations named PBs are deployed in an existing cellular network for recharging MTs via MPT wirelessly. Each PB is equipped with a solar panel and connected to grid by the CC, so PBs can be powered by hybrid power supplies. With the CC, PBs can get conventional energy from grid if the renewable power is insufficient, and achieve green energy cooperation.

In this work, we will develop effective power allocation optimization and green energy cooperation algorithm to improve the performance of cellular networks powered by hybrid energy sources. Our contributions can be summarized as follow:

- We propose a dual-level control architecture, in which controllers collect information for the CC, and the CC manage the entire system. The performance of the network can be improved by the dual-level control architecture.

- A low-complexity online water filling optimized power allocation strategy (WFOPAS) is proposed for maximizing the uplink rate. With the algorithm, the MT's average rate can be improved obviously. 
- We further propose a green energy and CSI aware scheme, that named water filling optimized green energy cooperation management strategy (WFOGECMS). With the scheme, the preference of the system can be improved. Moreover, the algorithm does not require statistical CSI and EH processes, which makes it applicable in unpredictable environment.

- Simulation results are provided to certify the theoretical analysis. It shows that, with renewable energy distributed unevenly, the WFOGECMS algorithm not only achieves the MT's average rate improvement, but also reduces the on-grid energy consumption.

The paper is organized as follows. Section 2 defines the system model. Section 3 formulates the uplink rate maximization problem. Section 4 proposes the WFOGECMS algorithm for the uplink rate maximization problem. Section 5 provides simulation results, and conclusions are presented in Section 6.

\section{System Model}

This section describes the definitions and assumptions of the network, and explains how the system deals with wireless power transfer and communication. The system model is illustrated in Fig. 1, and the notation is summarized in Table 1.
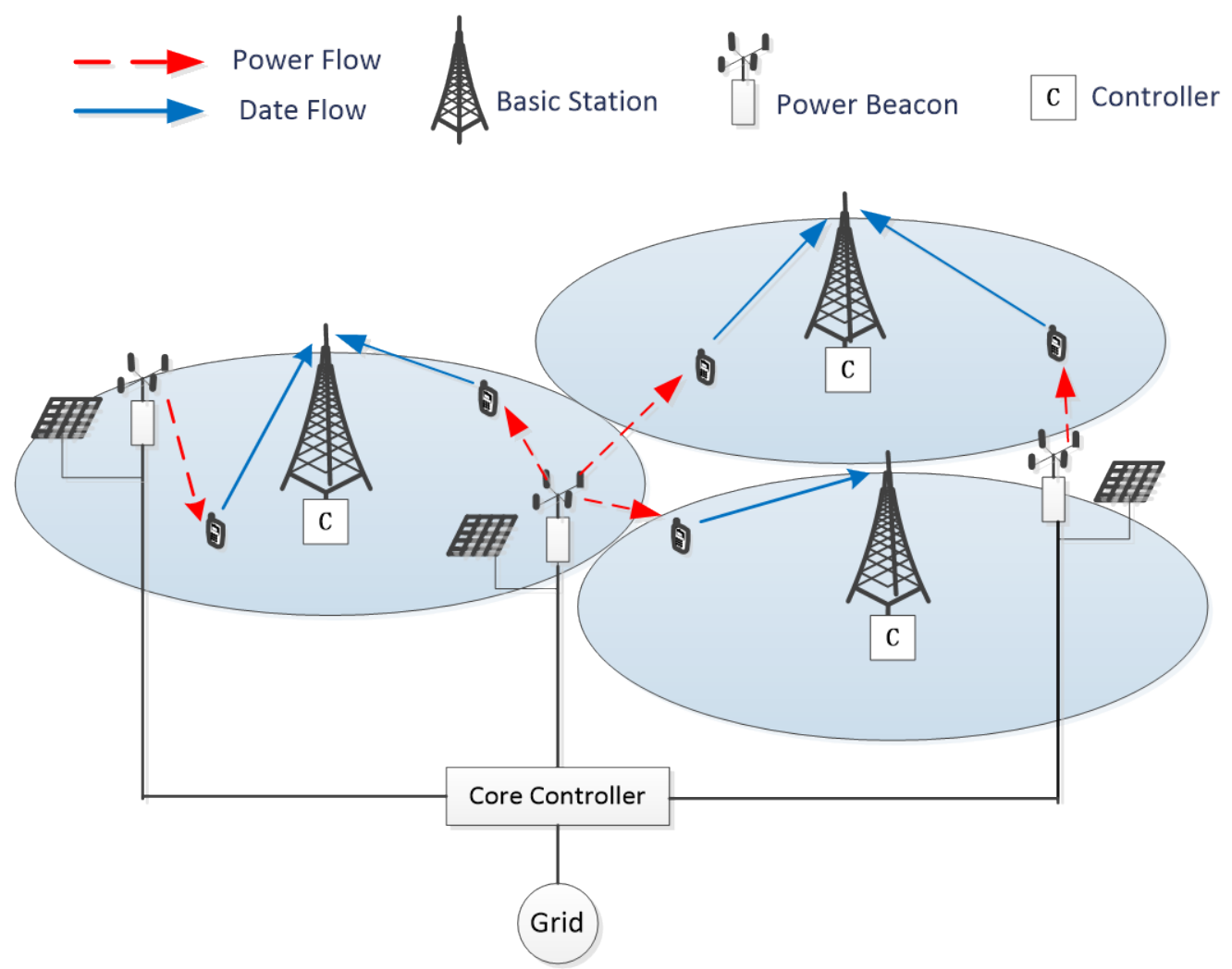

Fig. 1. System model

In our model, multiple base stations, PBs and MTs are independent with each other, and MTs have been divided into clusters corresponding to PBs. We focus on maximizing the 
uplink rate by designing the renewable energy and CSI aware scheme. In the dual-level control architecture, controllers are configured on base stations, gathering information relying on the base stations' coverage of the area. Information collected by controllers consists of: the position of PBs and MTs, the amount of harvested green energy, each MT's access status with PB and base station, and the CSI information. The CC, which connects to controllers (by its connection with base stations) and PBs by cables, responsible for complex calculation, and has a global grasp of the system. After analyzing the gathered data, the CC sends commands to PBs. According to the commands of the CC, PB can optimizes power allocation among its antennas, and green energy cooperation can be implemented by PBs locally injecting/drawing power into/from other PBs via the CC. The CC controls the direction of energy flow that exchange among PBs. Under the priority use of renewable energy, the shortage is supplemented by grid.

Furthermore, we assume para-static time-slotted model for both renewable energy and equivalent channels (there are two types of wireless channels in the network: the energy link and the data link, we collectively name them as the equivalent channel). The energy harvesting rate and the channel coefficient remain constant in each slot and may change from one slot to another. Without loss of generality, we choose one communication block as the reference time slot. We focus our study on one communication slot. In the following, we will present the energy model and the uplink cellular network model.

Table 1. Summary of notations

\begin{tabular}{|c|c|}
\hline Symbol & Meaning \\
\hline$N, M, S$ & The total number of PBs, MTs, base stations \\
\hline$E_{i}, M_{i}$ & The harvested renewable energy by $P B_{i}$, the number of MTs served by $P B_{i}$ \\
\hline Q & The total harvested renewable energy \\
\hline$p_{i}$ & The power consumption of $P B_{i}$ \\
\hline$p_{i, k}$ & The transmission power from the $P B_{i}$ to its k-th MT \\
\hline$E_{i}^{+}, E_{i}^{-}$ & The renewable energy drawn/injected by $P B_{i}$ \\
\hline$\zeta$ & Energy transfer efficiency \\
\hline$P_{i}^{G}$ & The consumed on-grid power by $P B_{i}$ \\
\hline$d, D$ & The Euclidian distance between MT and its PB, MT and its base station \\
\hline$U_{0}, P_{0}, B_{0}$ & The typical mobile terminal, base station and power beacon \\
\hline$q_{u_{0}}^{r}, q_{u_{0}}^{t}$ & The receiving, transmission power of the typical mobile terminal \\
\hline$q_{B_{0}}^{r}$ & The receiving power of the typical base station \\
\hline$\alpha, \beta$ & Path-loss exponent for a MPT channel, data channel \\
\hline$G_{\mathrm{m}}$ & The amplify factor in energy beamforming \\
\hline$\sigma^{2}$ & The white noise variance \\
\hline$\vartheta$ & The threshold of the signal to interference plus noise ratio \\
\hline$\varepsilon$ & The power sensitivity of energy harvest \\
\hline$f$ & The coefficient of channel fading \\
\hline $\mathrm{B}$ & The bandwidth of a base station \\
\hline
\end{tabular}




\subsection{The Energy Model}

We consider a system with $N$ PBs, $\boldsymbol{M}$ MTs, $S$ base stations. Each PB is equipped with $H$ ( $H \in Z \& H>1)$ antennas. Because of multiple antennas PB has, it can charge multiple target MTs simultaneously. PBs are distributed uniformly, and are independent with base stations and MTs. The number of MTs is no larger than the total number of PBs' antennas, i.e., $M \leq N H$. We denote the set of PBs and that of MTs as $\mathcal{N}=\{1, \ldots, N\}$ and $\mathcal{M}=\{1, \ldots, \mathbf{M}\}$, respectively.

As show in Fig. 1, PBs are powered by grid and green energy simultaneously. MT is powered by the nearest PB with energy beamforming. Base stations are powered by grid and just receive information from MTs. For convenience, we neglect the static energy consumption of PBs, base stations, MTs, controllers, and CC as [17] did. We consider the power management in $P \boldsymbol{B}_{\boldsymbol{i}}$ as shown in Fig. 2. BP uses renewable energy that is locally harvested or transferred from other PBs preferentially, otherwise, it consumes the on-grid power. The harvested renewable energy at $P B_{i}(i \in[1, \mathrm{~N}])$ is denoted by $E_{i}$, and

$$
\begin{aligned}
& \sum_{i=1}^{N} E_{i}=Q \\
& E_{i} \geq 0, \quad \forall i \in[1, \mathrm{~N}]
\end{aligned}
$$

Where $Q$ is the sum harvested renewable energy by PBs. We assume that $E_{1}, E_{2}, \ldots, E_{N}$ are independent and uniformly distributed in [0, Q], each with an equal mean of $Q / N$. Note that the independent energy distribution may correspond to the case in which the PBs are in different position.

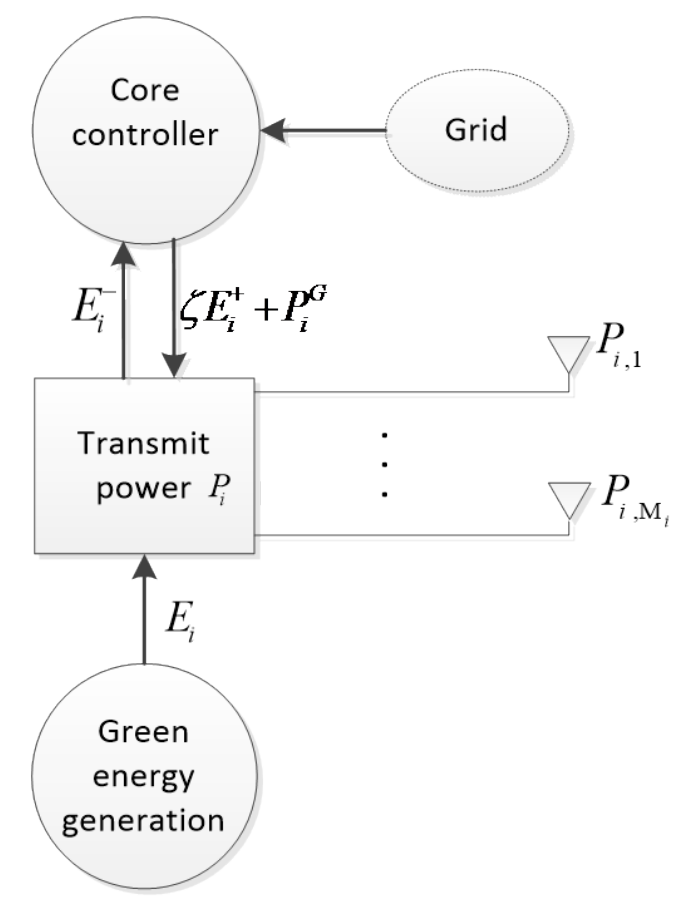

Fig. 2. Energy management schematics at $\boldsymbol{P} \boldsymbol{B}_{\boldsymbol{i}}$ 
The power of $P B_{i}$ consisted of four parts: the locally harvested renewable energy $E_{i}$, the drew/ injected green energy from/into other PBs via the CC $\zeta E_{i}^{+} / E_{i}^{-}$, and the power from grid $P_{i}^{G}$. $P B_{i}$ injected its locally harvested renewable energy into other PBs, only when its green energy is surplus. Set the number of MTs served by $P B_{i}$ is $M_{i}\left(M_{i} \in Z, M \geq M_{i} \geq 0\right)$, the power consumption of $P B_{i}$ can be given by

$$
P_{i}=\sum_{k=1}^{M_{i}} P_{i, k}=E_{i}+\zeta E_{i}^{+}-E_{i}^{-}+P_{i}^{G}
$$

where $\zeta \in[0,1]$ is the efficiency coefficient of energy transfer, and Eq. (3) should satisfy the following constraints:

$$
\begin{aligned}
& E_{i}^{+} E_{i}^{-}=0 \\
& E_{i}^{+} \geq 0, \forall i \in[1, \mathrm{~N}] \\
& E_{i}^{-} \geq 0, \quad \forall i \in[1, \mathrm{~N}] \\
& P_{i}^{G} \geq 0
\end{aligned}
$$

The proof of Eq. (4) is as follow.

By satisfying the constraints of Eq. (5) and Eq. (6), at least one of $E_{i}^{+}$and $E_{i}^{-}$is zero. Because in real-time applications only three cases will be happening to $P B_{i}$ :

$$
\begin{cases}E_{i}^{-}>0 \& E_{i}^{+}=0, & E_{i}-P_{i}>0 \\ E_{i}^{-}=0 \& E_{i}^{+}=0, & E_{i}-P_{i}=0 \\ E_{i}^{-}=0 \& E_{i}^{+}>0, & E_{i}-P_{i}<0\end{cases}
$$

Where $E_{i}-P_{i}>0, E_{i}-P_{i}=0$, and $E_{i}-P_{i}<0$ corresponding to three cases that the green energy of $P B_{i}$ overflowing, exactly right, inadequate respectively. Above all, we can get $E_{i}^{+} E_{i}^{-}=0$, and Eq. (4) is proved.

Without loss of generality, a typical MT is denoted by $U_{0}$, the nearest PB that serves $U_{0}$ is named the typical $\mathrm{PB}$ and denoted by $P B_{0}$. The energy link between $\mathrm{PB}$ and $\mathrm{MT}$ is characterized by path loss but no fading. The number of MTs served by $P B_{0}$ is $M_{0}\left(M_{0} \in Z, M \geq M_{0} \geq 0\right)$, assume $U_{0}$ is the k-th MT served by $P B_{0}\left(1 \leq k \leq M_{0}\right)$. The transmission power from $P B_{0}$ to $U_{0}$ is denoted by $P_{0, k}$, the received power of $U_{0}$ is $G_{m} P_{0, k} d_{0, k}{ }^{-\alpha}$, where $d_{0, k}$ is the Euclidian distance between $U_{0}$ and $P B_{0}$ in meter, $G_{m}$ is the amplify factor in energy beamforming, $\alpha>2$ is the path-loss exponent in energy link. Considering the power sensitivity of EH [30], we obtain the boundary condition Eq. (9) of the expression. So the received power of $U_{0}$ can be written as

$$
\begin{aligned}
& q_{u_{0}}^{r}=G_{m} P_{0, k}\left[\max \left(d_{0, k}, 1\right)\right]^{-\alpha} \\
& \text { s.t. } \quad G_{m} P_{0, k}\left[\max \left(d_{0, k}, 1\right)\right]^{-\alpha} \geq \varepsilon
\end{aligned}
$$


where $\varepsilon$ is the power sensitivity of $\mathrm{EH}$. (If we consider the side-lobe gain of other PBs, when one $\mathrm{PB}$ out of $P B_{0}$ denoted by $P B_{j}$ is working, it may have side-lobe gain to $U_{0}$. But the amplify factor of the side-lobe $G_{s}$ is almost one percent of $G_{m}$, meanwhile, the distance $d_{\mathrm{j}, \mathrm{k}}$ between $P B_{j}$ and $U_{0}$ is larger than $d_{0, k}$, so the interference of the side-lobe gain $G_{s} P_{\mathrm{j}, k}\left[\max \left(d_{\mathrm{j}, k}, 1\right)\right]^{-\alpha}$ is much less than the main-lobe that we ignore it).

We suppose the efficiency factor of energy conversion is equal to one (ideal). Thus the transmission power of $U_{0}$ can be given as

$$
q_{u_{0}}^{t}=q_{u_{0}}^{r}
$$

\subsection{The Uplink cellular network Model}

In our model, base stations and MTs are distributed uniformly. MTs in the same cell are independent and the carriers with the corresponding base station are orthographical to each other, the environment is interference-limited. The nearest base station serves $U_{0}$ is named the typical base station and denoted by $B_{0}$. The date link is characterised by pass-loss and fading, and the channel fading $f$ is assumed to follow exponential distribution (Rayleigh fading), i.e. $f \sim \exp (1)$. Let $U_{0}$ sends data to $B_{0}$ with power $q_{u_{0}}^{t}$, the received signal by $B_{0}$ is $q_{B_{0}}^{r}=q_{u_{0}}^{t} f_{0} D_{0,0}^{-\beta}$, where $D_{0,0}$ is the Euclidian distance between $U_{0}$ and $B_{0}$ in meter, and $\beta>2$ is the path-loss exponent. It can be expressed as

$$
q_{B_{0}}^{r}=G_{m} P_{0, k} f_{0}\left[\max \left(d_{0, k}, 1\right)\right]^{-\alpha} \cdot\left[\max \left(D_{0,0}, 1\right)\right]^{-\beta}
$$

The interference comes from MTs out of $U_{B_{0}}$ :

$$
I_{0}=\sum_{M_{v} \in\left(M-U_{B_{0}}\right)} G_{m} P_{v} f_{v}\left[\max \left(d_{v}, 1\right)\right]^{-\alpha} \cdot\left[\max \left(D_{v}, 1\right)\right]^{-\beta}
$$

Where $M_{v}$ is the v-th $\left(v \in\left[1, \mathrm{M}-\mathrm{nu}_{0}\right]\right)$ interfering MT to the data link between $U_{0}$ and $B_{0}$. $U_{B_{0}}$ is the MTs set that served by $B_{0}, n_{0}$ is the number of MTs within $U_{B_{0}} . P_{v}$ is the transmission power from PB to $M_{v}, d_{v}$ is the Euclidian distance between PB and $M_{v}, D_{v}$ is the Euclidian distance between $B_{0}$ and $M_{v}$.

The SINR of the data link is $\operatorname{SINR}_{0}=\frac{q_{B_{0}}^{r}}{I_{0}+\sigma^{2}}$, in which $\sigma^{2}$ is the background additive white Gaussian noise (AWGN) variance. The rate of the data link from $U_{0}$ to $B_{0}$ is given by

$$
R_{0}=\frac{B}{n u_{0}} \log _{2}\left(1+\frac{G_{m} P_{0, k} f_{0}\left[\max \left(d_{0, k}, 1\right)\right]^{-\alpha} \cdot\left[\max \left(D_{0, k}, 1\right)\right]^{-\beta}}{\sum_{M_{v} \in\left(M-U_{B_{0}}\right)} G_{m} P_{v} f_{v}\left[\max \left(d_{v}, 1\right)\right]^{-\alpha} \cdot\left[\max \left(D_{v}, 1\right)\right]^{-\beta}+\sigma^{2}}\right)
$$

Where $B$ is the bandwidth of the base station. 


\section{Problem Formulation}

In this section, we first introduce the uplink rate maximization problem, then solve the problem with the WFOPAS algorithm.

\subsection{The Uplink Rate Maximization Problem}

Considering the energy side, $M$ MTs have been divided into clusters corresponding to PBs. The number of MTs served by $P B_{i}$ is $M_{i}\left(\sum_{i=1}^{N} M_{i}=M\right)$. Assume the k-th $\left(1 \leq k \leq M_{i}\right)$ MT served by $P B_{i}$ is served by $\mathrm{BS}_{j}$, with Eq. (13), The sum-rate of the i-th MT cluster is $\sum_{k=1}^{M_{i}} \frac{B}{n u_{j}} \log _{2}\left(1+\frac{G_{m} P_{\mathrm{i}, \mathrm{k}} f_{\mathrm{j}, \mathrm{l}}\left[\max \left(d_{\mathrm{i}, \mathrm{k}}, 1\right)\right]^{-\alpha} \cdot\left[\max \left(D_{\mathrm{j}, 1}, 1\right)\right]^{-\beta}}{\sum_{M_{v} \in\left(M-U_{B_{j}}\right)} G_{m} P_{v} f_{v}\left[\max \left(d_{v}, 1\right)\right]^{-\alpha} \cdot\left[\max \left(D_{v}, 1\right)\right]^{-\beta}+\sigma^{2}}\right)$. We will use the Uplink Rate (UR) as the performance metric, which is the MT's average rate and defined as $U R=\frac{1}{M} \sum_{i=1}^{N} \sum_{k=1}^{M_{i}} \frac{B}{n u_{j}} \log _{2}\left(1+\frac{G_{m} P_{\mathrm{i}, k} f_{\mathrm{j}, \mathrm{l}}\left[\max \left(d_{\mathrm{i}, k}, 1\right)\right]^{-\alpha} \cdot\left[\max \left(D_{\mathrm{j}, 1}, 1\right)\right]^{-\beta}}{\sum_{M_{v} \in\left(M-U_{B_{j}}\right)} G_{m} P_{v} f_{v}\left[\max \left(d_{v}, 1\right)\right]^{-\alpha} \cdot\left[\max \left(D_{v}, 1\right)\right]^{-\beta}+\sigma^{2}}\right)$

To minimize the on-grid energy consumption, consider the situation that the system powered by renewable energy only, and green energy cooperation has not been implemented yet. Consequently, the uplink rate maximization problem can be formulated as $P 1$ :

$$
\begin{aligned}
& \max _{\left\{P_{i, k}\right\}} \frac{B}{M} \sum_{i=1}^{N} \sum_{k=1}^{M_{i}} \frac{1}{n u_{j}} \log _{2}\left(1+\frac{G_{m} P_{\mathrm{i}, k} f_{\mathrm{j}, 1}\left[\max \left(d_{\mathrm{i}, k}, 1\right)\right]^{-\alpha} \cdot\left[\max \left(D_{\mathrm{j}, 1}, 1\right)\right]^{-\beta}}{\sum_{M_{v} \in\left(M-U_{B_{j}}\right)} G_{m} P_{v} f_{v}\left[\max \left(d_{v}, 1\right)\right]^{-\alpha} \cdot\left[\max \left(D_{v}, 1\right)\right]^{-\beta}+\sigma^{2}}\right) \\
& \text { s.t. } \quad \sum_{k=1}^{M_{i}} P_{i, k} \leq E_{i} \quad \forall i \in[1, \mathrm{~N}] \\
& \quad P_{i, k} \geq 0 \quad \forall i \in[1, \mathrm{~N}], \forall k \in\left[1, \mathrm{M}_{i}\right]
\end{aligned}
$$

In P1, the peak summed transmit power constraint and the power non-negativity constraint are imposed in Eq. (16) and Eq. (17), respectively.

Since $M, N, B, n u_{j}, M_{i}$ is given, when the sum-rate of each cluster is maximum so as the sum-rate of the network. Thus, P1 can be decomposed to maximize the rate of each cluster, so we equivalently introduce a modified version of P1 as follows:

$$
\frac{B}{M} \sum_{i=1}^{N}\left(\max _{\left\{P_{1, k}\right\}} \sum_{k=1}^{M_{i}} \frac{1}{n u_{j}} \log _{2}\left(1+\frac{G_{m} P_{\mathrm{i}, k} f_{\mathrm{j}, 1}\left[\max \left(d_{\mathrm{i}, k}, 1\right)\right]^{-\alpha} \cdot\left[\max \left(D_{\mathrm{j}, 1}, 1\right)\right]^{-\beta}}{\sum_{M_{v} \in\left(M-U_{B_{j}}\right)} G_{m} P_{v} f_{v}\left[\max \left(d_{v}, 1\right)\right]^{-\alpha} \cdot\left[\max \left(D_{v}, 1\right)\right]^{-\beta}+\sigma^{2}}\right)\right)
$$


s.t. (16), (17)

and the version of $\mathrm{P} 2$ is expressed as:

$P 2$ :

$$
\max _{\left\{\mathrm{P}_{\mathrm{i}, k}\right\}} \sum_{k=1}^{M_{i}} \log _{2}\left(1+\frac{G_{m} P_{\mathrm{i}, k} f_{\mathrm{j}, 1}\left[\max \left(d_{\mathrm{i}, k}, 1\right)\right]^{-\alpha} \cdot\left[\max \left(D_{\mathrm{j}, 1}, 1\right)\right]^{-\beta}}{\sum_{M_{v} \in\left(M-U_{B_{j}}\right)} G_{m} P_{v} f_{v}\left[\max \left(d_{v}, 1\right)\right]^{-\alpha} \cdot\left[\max \left(D_{v}, 1\right)\right]^{-\beta}+\sigma^{2}}\right)
$$

s.t. (16), (17)

Hence, let $i=1,2, \ldots, N \mathrm{P} 1$ is the sum of $\mathrm{P} 2$, any feasible solution for $\mathrm{P} 2$ is also feasible for P1.

\subsection{The WFOPAS Algorithm}

We solve problem P2 for the optimal power allocation solution among each cluster' MTs. It can be verified that $\mathrm{P} 2$ is a convex problem since the objective function is concave. At the same time, all the constraints are linear. Thus, the optimal solution satisfies the KKT conditions, and the Lagrange duality method can be applied to solve this problem optimally [31].

Let $\varphi_{i} \geq 0, b_{i, k} \geq 0, \forall i \in[1, \mathrm{~N}], \forall k \in\left[1, \mathrm{M}_{i}\right]$ be the Lagrange operators associated with each of the power constraints of P2 given in Eq. (16) and Eq. (17). Then, the Lagrange function of $\mathrm{P} 2$ can be defined as

$$
\begin{aligned}
& L_{i}\left(\left\{P_{\mathrm{i}, k}\right\},\left\{\varphi_{\mathrm{i}}\right\},\left\{\mathrm{b}_{\mathrm{i}, k}\right\}\right) \\
& \quad=\sum_{k=1}^{M_{i}} \log _{2}\left(1+\frac{G_{m} P_{\mathrm{i}, k} f_{\mathrm{j}, \mathrm{l}}\left[\max \left(d_{\mathrm{i}, k}, 1\right)\right]^{-\alpha} \cdot\left[\max \left(D_{\mathrm{j}, 1}, 1\right)\right]^{-\beta}}{\sum_{M_{v} \in\left(M-U_{B_{j}}\right)} G_{m} P_{v} f_{v}\left[\max \left(d_{v}, 1\right)\right]^{-\alpha} \cdot\left[\max \left(D_{v}, 1\right)\right]^{-\beta}+\sigma^{2}}\right)-\varphi_{i}\left(\sum_{k=1}^{M_{i}} P_{i, k}-E_{i}\right)+\sum_{k=1}^{M_{i}} b_{i, k} P_{i, k}
\end{aligned}
$$

where $\varphi_{i}, b_{i, k}$ meet the mutual slack conditions, which is

$$
\begin{aligned}
& \varphi_{i}\left(\sum_{k=1}^{M_{i}} P_{i, k}-E_{i}\right)=0, i \in[1, \mathrm{~N}] \\
& b_{i, k} P_{i, k}=0 \quad k \in\left[1, \mathrm{M}_{i}\right]
\end{aligned}
$$

thus, we can solve P2 by solving Eq. (20) equivalently. Solving $\partial L_{i} / \partial P_{i, k}=0$, the optimal power allocation $p_{i, k}^{*}$ can be expressed as a function of Lagrange multipliers

$$
p_{i, k}^{*}=\frac{1}{\varphi_{i}-b_{i, k}}-\frac{\sum_{M_{v} \in\left(M-U_{B_{j}}\right)} G_{m} f_{v}\left[\max \left(d_{v}, 1\right)\right]^{-\alpha} \cdot\left[\max \left(D_{v}, 1\right)\right]^{-\beta}+\sigma^{2}}{G_{m} f_{\mathrm{j}, 1}\left[\max \left(d_{\mathrm{i}, k}, 1\right)\right]^{-\alpha} \cdot\left[\max \left(D_{\mathrm{j}, 1}, 1\right)\right]^{-\beta}}
$$

When the system powered by renewable energy only, and green energy cooperation has not been implemented yet. The green energy has been used up to maximize the sum-rate of the network, it is easy to obtain $P_{i}^{G}=0$ and $\sum_{k=1}^{M_{i}} P_{i, k}=E_{i}$, so $\sum_{k=1}^{M_{i}} P_{i, k}-E_{i}=0$. With Eq. (21) the Lagrange operator $\varphi_{i}$ can equal to any nonnegative value. With Eq. (17) and Eq. (22), it can 
be easily verified that $b_{i, k}=0$. Thus, the power distribution reaches a fixed level $1 / \varphi_{i}$, the optimal solution can be obtained as

$$
\begin{aligned}
& p_{i, k}^{*}=\max \left\{\left(\frac{1}{\varphi_{i}}-\frac{\sum_{M_{v} \in\left(M-U_{B_{j}}\right)} G_{m} f_{v}\left[\max \left(d_{v}, 1\right)\right]^{-\alpha} \cdot\left[\max \left(D_{v}, 1\right)\right]^{-\beta}+\sigma^{2}}{G_{m} f_{\mathrm{j}, \mathrm{l}}\left[\max \left(d_{\mathrm{i}, k}, 1\right)\right]^{-\alpha} \cdot\left[\max \left(D_{\mathrm{j}, 1}, 1\right)\right]^{-\beta}}\right), 0\right\} \\
& \text { s.t. } \sum_{k=1}^{M_{i}} P_{i, k}=E_{i} \quad \forall i \in[1, \mathrm{~N}], \forall k \in\left[1, \mathrm{M}_{i}\right]
\end{aligned}
$$

And how to obtain $p_{i, k}^{*}$ is given in the WFOPAS algorithm as shown in Table 2.

Table 2. Algorithm 1: The WFOPAS algorithm for Solving Problem P2

At the beginning initialize $i=1$.

1: Obtain $E_{i}, M_{i}, G_{m}, d_{\mathrm{i}, k}, D_{\mathrm{j}, 1}, \sigma^{2}\left(k=1, \ldots, \mathrm{M}_{i}\right)$ from the CC.

2: Initialize the iteration factor $S=1$,

a) calculating

$$
\lambda_{i}=\frac{1}{\varphi_{i}}=\frac{E_{i}}{M_{i}-s+1}\left[1+\sum_{i=1}^{M_{i}-s+1} \frac{\sum_{M_{v} \in\left(M-U_{B_{j}}\right)} G_{m} f_{v}\left[\max \left(d_{v}, 1\right)\right]^{-\alpha} \cdot\left[\max \left(D_{v}, 1\right)\right]^{-\beta}+\sigma^{2}}{G_{m} f_{\mathrm{j}, 1}\left[\max \left(d_{\mathrm{i}, k}, 1\right)\right]^{-\alpha} \cdot\left[\max \left(D_{\mathrm{j}, 1}, 1\right)\right]^{-\beta}}\right]
$$

b) Calculating

$$
\begin{gathered}
\sum_{i, k}=\lambda_{i}-E_{i} \cdot \frac{\sum_{M_{v} \in\left[M-U_{B_{j}}\right.} G_{m} f_{v}\left[\max \left(d_{v}, 1\right)\right]^{-\alpha} \cdot\left[\max \left(D_{v}, 1\right)\right]^{-\beta}+\sigma^{2}}{G_{m} f_{\mathrm{j}, \mathrm{l}}\left[\max \left(d_{\mathrm{i}, k}, 1\right)\right]^{-\alpha} \cdot\left[\max \left(D_{\mathrm{j}, 1}, 1\right)\right]^{-\beta}}, k=1, \ldots, M_{i}-s+1 \\
\text { c) If } P_{i, k}<0, \forall k \text {, set } p_{i, M_{i}-s+1}=0, s=s+1 \text {, turn to step (a). }
\end{gathered}
$$

3: Until $\forall P_{i, k} \geq 0,\left(k=1, \ldots, \mathrm{M}_{i}\right)$, the optimal power allocation scheme for the i-th cluster' MTs has been obtained.

4: Set $i=i+1$, turn to step 1 .

Until $\forall P_{\mathrm{i}, k} \geq 0,\left(\mathrm{i}=\mathrm{N}, k=1, \ldots, \mathrm{M}_{N}\right)$, break.

As a result, we obtain $\left\{P_{i, k}^{*}\right\}$ for all MTs finally, that maximize the sum-rate of the network. Thus P2 have been solved completely.

In the following, we compare the performance of the proposed WFOPAS algorithm and the average power allocation strategy (APAS) used in [12] in a system powered by green energy only, and no green energy cooperation among PBs. During comparing, we consider a two-cell simple network. We set $N=2$ PBs, $S=2$ base stations, $M=3$ MTs. The path-loss exponents are $\alpha=3$ and $\beta=3$. We assume the background AWGN variance in every data link are the same and equal to $-110 \mathrm{dBm}$. The amplify factor in MPT beamforming is $G_{m}=10$ [12]. For simplification, the bandwidth of base station $B=1 \mathrm{~Hz}$. The radius of the small cell cellular network is $R=500$ meter, simulating 100000 times.

In Fig. 3, it is visible that the MT's average rate increases with the total harvested renewable energy. Under different SINR threshold $\vartheta$, the performance with WFOPAS is much better than that of APAS. The MT's average rate with WFOPAS is improved about 
7\% 20\% than APAS. This behavior can be explained as follows. With WFOPAS, the transmission power in every PB can be allocated adaptively based on the CSI. In each cluster, more power is distributed to the channels whose CSI are good, and less energy is allocated to the MTs with poor channel conditions. But with APAS, power allocation can't be optimized.

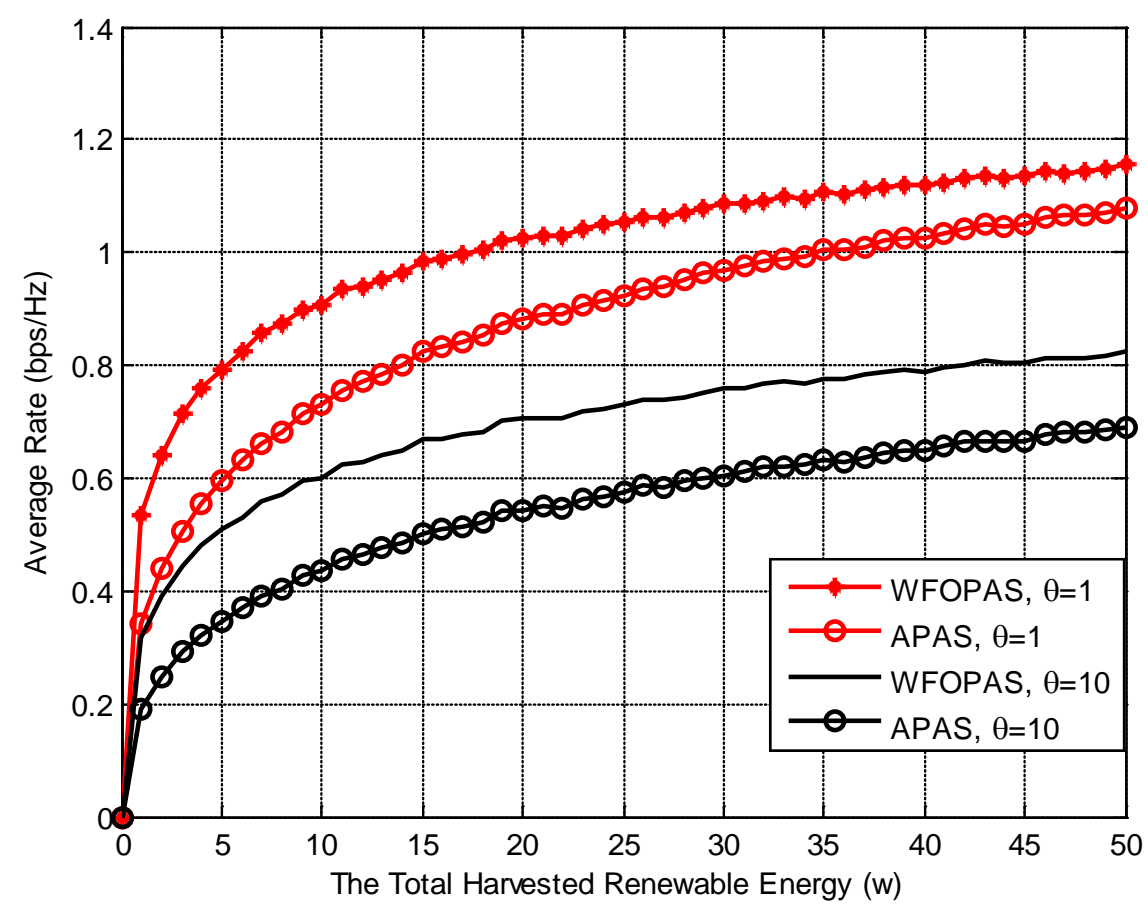

Fig. 3. Average rate VS The total harvested renewable energy

\section{The Water Filling Optimized Green Energy Cooperation Management Strategy}

So far, we have solved P2, and the WFOPAS algorithm can optimize the MT's average rate of the system. In the following, we focus our study on maximize the rate of the system by use green energy cooperation and WFOPAS algorithm jointly in cellular networks, which powered by green energy and grid together. We will show how to implement green energy cooperation with low complexity.

The difference between WFOPAS and WFOGECMS is focused on two aspects: one is whether adopt green energy cooperation, and the other is the range of the water filling optimization algorithm applied. In the WFOPAS algorithm, green energy has no cooperation, the algorithm is applied in each MT cluster corresponding to $\mathrm{PB}$, and it explains how to allocate $E_{i}$ among antennas within $P B_{i}$. In the WFOGECMS algorithm, green energy cooperation carried out via the CC, and the algorithm is implemented in all MTs of the network at the same time. The WFOGECMS algorithm explains how to allocate $Q$ among all MTs. 


\subsection{The WFOGECMS Algorithm}

Green energy cooperation can be implemented by PBs with extra/ inadequate renewable energy locally injecting/drawing power into/from other PBs via the CC. The direction of the energy flow between the CC and PB is controlled by the CC. The harvested renewable energy is efficiently utilized to maximize the rate, and energy shortage is supplied by grid. The WFOGECMS algorithm is expressed in Table 3. Whenever the CSI or $\forall E_{i}$ changed, the CC recalculates and adjusts the parameters of the system immediately. And the rate of the system can be optimized by the WFOGECMS algorithm.

Table 3. Algorithm 2: The WFOGECMS Algorithm

Whenever CSI or $\forall E_{i}$ changed, turn to step 1.

1: MTs select the nearest PB and base station to build pre-access.

2: Controllers collect information such as: the position of MTs', PBs' and base stations'; the harvested renewable energy $E_{i}$ in every PB; the access situation of each MT to base station and PB; and the CSI. Then controllers upload the information to the CC.

3: According to the collected data, the CC calculates $P_{i}(i=1,2, \ldots N)$ for each PB.

4: With $P_{\mathrm{i}}$ and $E_{i}$, the CC calculates the energy status $\Delta E_{i}=E_{i}-P_{\mathrm{i}}$ in every PB, and affixes each PB with a green $\left(\Delta E_{i}>0\right)$, white $\left(\Delta E_{i}=0\right)$, or red $\left(\Delta E_{i}<0\right)$ label.

5: PBs with green/red label inject/draw extra/ inadequate renewable energy into/from other PBs via the CC, and PBs with red label obtain the required green energy from the CC. The shortage is complemented by grid.

6: MTs charged by PBs and communicate with base stations until the end of the current round, turn to step 1.

\subsection{The Mathematic Model of WFOGECMS}

In the hybrid cellular network, renewable energy and grid co-exist to power PBs. The CC can get $d_{j}$ (the distance between $M T_{j}$ and its nearest $\mathrm{PB}$ ), $D_{j}$ (the distance between $M T_{j}$ and its nearest base station $\left.B S_{i}\right) \quad j \in[1, \mathrm{M}]$, and the total harvested green energy $Q$ from the system. The renewable energy is utilized efficiently to maximize the rate of the system. Consequently, the uplink rate maximization problem can be formulated as

P3:

$$
\begin{array}{ll}
\max _{\left\{P_{j}\right\}} & \frac{1}{M} \sum_{j=1}^{M} \frac{B}{n u_{i}} \log _{2}\left(1+\frac{G_{m} P_{j} f_{\mathrm{j}}\left[\max \left(d_{j}, 1\right)\right]^{-\alpha} \cdot\left[\max \left(\mathrm{D}_{j}, 1\right)\right]^{-\beta}}{\sum_{M_{v} \in\left(M-U_{B_{i}}\right)} G_{m} P_{v} f_{v}\left[\max \left(d_{v}, 1\right)\right]^{-\alpha} \cdot\left[\max \left(D_{v}, 1\right)\right]^{-\beta}+\sigma^{2}}\right) \\
\text { s.t. } & \sum_{j=1}^{M} P_{j} \leq Q \\
& P_{j} \geq 0 \quad \forall j \in[1, \mathrm{M}]
\end{array}
$$

where $P_{j}$ is the transmission power from PB to $M T_{j}$. We solve problem P3 for the optimal power allocation solution. Similar to P2, the Lagrange duality method can be applied to solve P3 optimally. 
Let $\varphi \geq 0, b_{j} \geq 0, \forall j \in[1, \mathrm{M}]$ be the Lagrange operators associated with each of the $\mathrm{M}$ power constraints of problem P3 given in Eq. (27) and Eq. (28). Since $M, B, n u_{i}$ is given, the Lagrange function of $\mathrm{P} 3$ can be defined as

$$
\begin{aligned}
& L\left(\left\{P_{j}\right\},\{\varphi\},\left\{\mathrm{b}_{j}\right\}\right) \\
& \quad=\sum_{j=1}^{M} \log _{2}\left(1+\frac{G_{m} P_{j} f_{\mathrm{j}}\left[\max \left(d_{j}, 1\right)\right]^{-\alpha} \cdot\left[\max \left(\mathrm{D}_{j}, 1\right)\right]^{-\beta}}{\sum_{M_{v} \in\left(M-U_{B_{i}}\right)} G_{m} P_{v} f_{v}\left[\max \left(d_{v}, 1\right)\right]^{-\alpha} \cdot\left[\max \left(D_{v}, 1\right)\right]^{-\beta}+\sigma^{2}}\right)-\varphi\left(\sum_{j=1}^{M} P_{j}-Q\right)+\sum_{j=1}^{M} b_{j} P_{j}
\end{aligned}
$$

where $\varphi, b_{j}$ meet the mutual slack conditions, which is

$$
\begin{aligned}
& \varphi\left(\sum_{j=1}^{M} P_{j}-Q\right)=0, \forall j \in[1, \mathrm{M}] \\
& b_{j} P_{j}=0 \quad \forall j \in[1, \mathrm{M}]
\end{aligned}
$$

Thus, we can solve P3 by solving Eq. (29) equivalently. Solving KKT conditions Lagrange operators $\partial L / \partial P_{j}=0$, the optimal power allocation $p_{i}^{*}$ can be expressed as a function of Lagrange multipliers

$$
p_{j}^{*}=\frac{1}{\varphi-b_{j}}-\frac{\sum_{M_{v} \in\left(M-U_{B_{i}}\right)} G_{m} f_{v}\left[\max \left(d_{v}, 1\right)\right]^{-\alpha} \cdot\left[\max \left(D_{v}, 1\right)\right]^{-\beta}+\sigma^{2}}{G_{m} f_{j}\left[\max \left(d_{j}, 1\right)\right]^{-\alpha} \cdot\left[\max \left(\mathrm{D}_{j}, 1\right)\right]^{-\beta}}
$$

When the system powered by renewable energy only, and the green energy has been used up to maximize the rate of the network, it is easy to obtain $\sum_{j=1}^{M} P_{j}=Q$, with Eq. (30) the Lagrange operator $\varphi$ can equal to any nonnegative value. With Eq. (28) and Eq. (31) it can be easily verified that $b_{j}=0$. Thus, the power distribution reaches a fixed level $1 / \varphi$, the optimal solutions can be obtained as

$$
\begin{aligned}
& p_{j}^{*}=\max \left\{\left(\frac{1}{\varphi}-\frac{\sum_{M_{v} \in\left(M-U_{B_{i}}\right)} G_{m} f_{v}\left[\max \left(d_{v}, 1\right)\right]^{-\alpha} \cdot\left[\max \left(D_{v}, 1\right)\right]^{-\beta}+\sigma^{2}}{G_{m} f_{j}\left[\max \left(d_{j}, 1\right)\right]^{-\alpha} \cdot\left[\max \left(\mathrm{D}_{j}, 1\right)\right]^{-\beta}}\right), 0\right\} \\
& \text { s.t. } \sum_{j=1}^{M} P_{j}=Q \quad \forall j \in[1, \mathrm{M}]
\end{aligned}
$$

How to obtain $p_{j}^{*}$ is given in the WFOGECMS algorithm as shown in Table 4.

As a result, we have finally obtained $\left\{P_{j}^{*}\right\}$ for all MTs, which makes the average rate of network maximal. Thus P3 have been solved completely.

According to Table 3, the CC calculates each PB's total transmission power $P_{i}$ by summing $P_{j}$ within the same cluster, then jointing $E_{i}$ calculates $\Delta E_{i}$. After that, commands are sent to PBs. At last, PBs inject/draw redundant/scanty green energy into/from other PBs via the CC to implement green energy cooperation. 
Table 4. Algorithm 3: The WFOGECMS Algorithm for Solving Problem P3

At the beginning initialize $i=1$.

1: Obtain $Q, G_{m}, d_{j}, D_{j}, \sigma^{2}(\mathrm{j}=1,2, \ldots, \mathrm{M})$ from the system.

2: Initialize the iterations factor $\mathrm{s}=1$,

a) calculating

$$
\lambda=\frac{1}{\varphi}=\frac{Q}{M-s+1}\left[1+\sum_{j=1}^{M-s+1} \frac{\sum_{M_{v} \in\left(M-U_{B_{i}}\right)} G_{m} f_{v}\left[\max \left(d_{v}, 1\right)\right]^{-\alpha} \cdot\left[\max \left(D_{v}, 1\right)\right]^{-\beta}+\sigma^{2}}{G_{m} f_{j}\left[\max \left(d_{j}, 1\right)\right]^{-\alpha} \cdot\left[\max \left(D_{j}, 1\right)\right]^{-\beta}}\right]
$$

b) Calculating

$$
\begin{gathered}
P_{j}=\lambda-Q . \frac{\sum_{M_{v} \in\left(M-U_{B_{i}}\right)} G_{m} f_{v}\left[\max \left(d_{v}, 1\right)\right]^{-\alpha} \cdot\left[\max \left(D_{v}, 1\right)\right]^{-\beta}+\sigma^{2}}{G_{m} f_{j}\left[\max \left(d_{j}, 1\right)\right]^{-\alpha} \cdot\left[\max \left(D_{j}, 1\right)\right]^{-\beta}}, j=1,2, \ldots, M-s+1 \\
\text { c) } \quad \text { If } P_{j}<0, \forall j \in[1, \mathrm{M}] \text {, set } p_{M-s+1}=0, s=s+1 \text {, turn to step (a). }
\end{gathered}
$$

Until $\forall p_{j} \geq 0$, the optimal power allocation scheme for all MTs has been obtained.

In the following, we will show the performance of the water level $\lambda$ in the WFOGECMS algorithm. The results come from calculate. Suppose that there are $N=10 \mathrm{PBs}$, and $S=10$ base stations in the system. The other parameters are the same as before: $\alpha=3, \beta=3$, $G_{m}=10$ [12], $R=500$ meter, $\mathrm{B}=1 \mathrm{~Hz}$, simulation 10000 times. The background AWGN variance in every data link are the same and equal to $-110 \mathrm{dBm}$. In Fig. 4, the performance of $\lambda=1 / \varphi$ is presented, which is the water level in the WFOGECMS algorithm. It is visible that $\lambda$ increases with $\mathrm{Q}$, and decreases with the density of MTs. The smaller the density of MTs and the larger the total harvested green energy, the higher the water level will be.

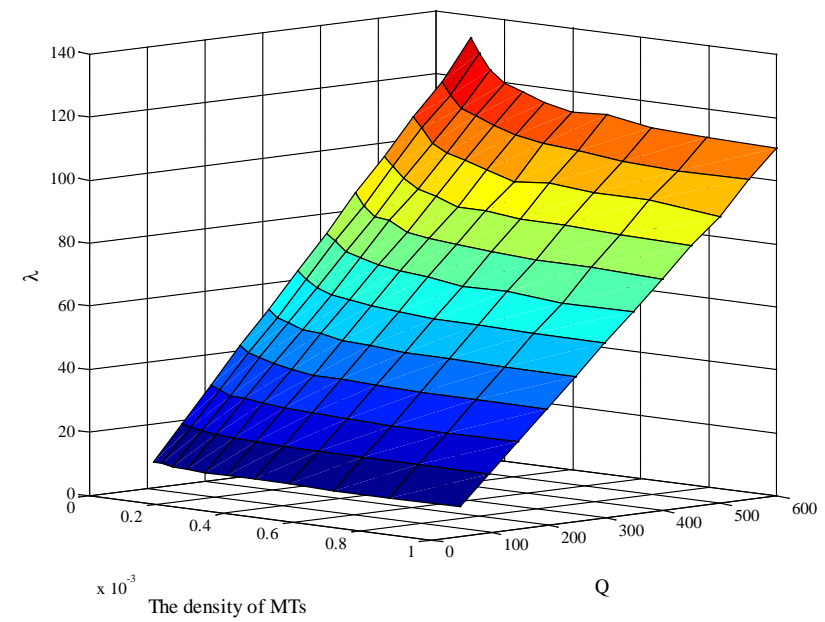

Fig. 4. The performance of $\lambda$ VS the density of MTs and Q 


\section{Simulation Results}

In this section, we will verify the theoretical results derived in Section 4 and evaluate the performance of the proposed WFOGECMS algorithm by simulation.

First, we consider a cellular network with $N=10$ PBs, $S=10$ base stations, $M=30$ MTs unless otherwise specified. In addition, $\alpha=3, \beta=3, G_{m}=10$ [12], $\vartheta=1, \zeta=0.9$ [17], $R=500$ meter. For simplification, $\mathrm{B}=1 \mathrm{~Hz}$, and the background noise in each data link is $-110 \mathrm{dBm}$, simulation 1000000 times.

Fig. 5 shows the MT's average rate of the system. With the practical energy transfer efficiency $\zeta=0.9$, the average rate increases with the total harvested renewable energy. It is visible that, with the proposed WFOGECMS algorithm, that 'Joint WFOPAS and green energy cooperation', the average rate is the best among 'Joint APAS and green energy cooperation' scheme and 'No green energy cooperation, WFOPAS only' scheme that given in section 3.2. It is also observed that, when $Q$ is little (smaller than 100 watt), the gap between them is large; with the increase of Q, the gap is getting smaller. It means when the total harvested renewable energy is big enough, the improvement brought by WFOGECMS algorithm is no longer obvious. Thereby, when the total harvested renewable energy is not enough, the WFOGECMS algorithm can bring a significant improvement. Third, the employment of green energy cooperation can bring up to 10\% promotion in the MT's average rate than without it, while WFOPAS can get 5.4\% improvement than APAS, the benefit with green energy cooperation is more obvious. And we can obtain that the gain of green energy cooperation is more dominant over that of WFOPAS, and "Joint WFOPAS and green energy cooperation" strategy, whose name is WFOGECMS, is optimal.

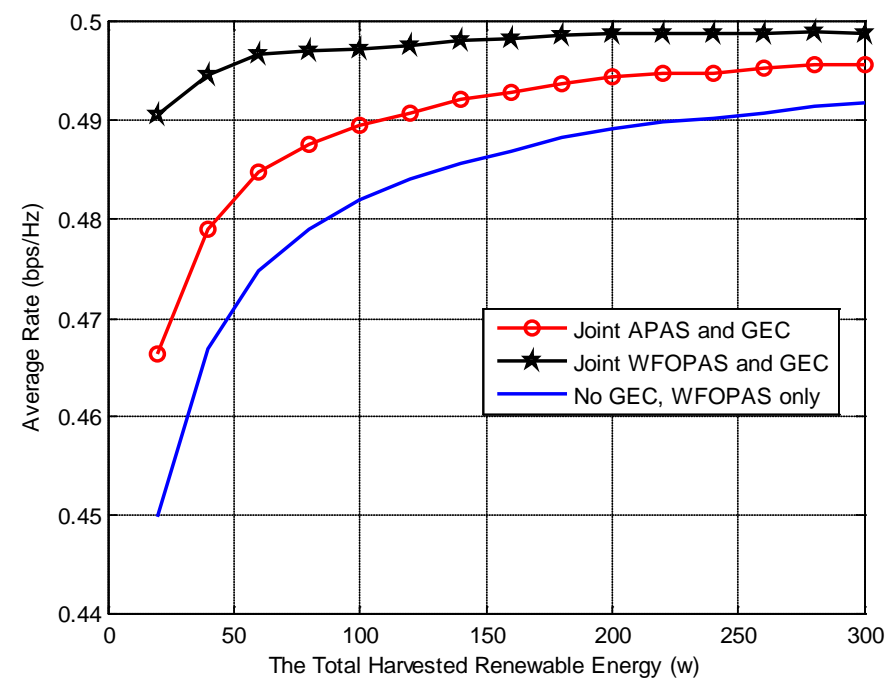

Fig. 5. The average rate vs $Q$

Next, we evaluate the performance of the proposed WFOGECMS algorithm and another suboptimal average power allocation strategy with green energy cooperation (APASGEC) scheme in a practical system with $\mathrm{N}=10 \mathrm{PBs}, \mathrm{S}=10$ base stations, and $\mathrm{M}=30 \mathrm{MTs}$. In our simulation, we assume that all PBs are deployed in different position with different renewable energy generation capacities. As shown in Fig. 4, the water level $\lambda$ in the water filling 
optimization algorithm is described. In Fig. 6, we show the network's sum-rate and on-grid energy consumption performance. It is observed that the WFOGECMS algorithm with $\zeta=0.9$ is better than APASGEC scheme; as the total harvested green energy Q increases, especially after $\mathrm{Q}=200$ watt, the gap between them becomes smaller quickly. Which shows little gain in the sum-rate and approximate $20 \%$ decrease of on-grid energy consumption with WFOGECMS. The improvement of the performance mainly because the CC adjusts the renewable energy allocation among PBs dynamically and employ green energy cooperation adaptively.

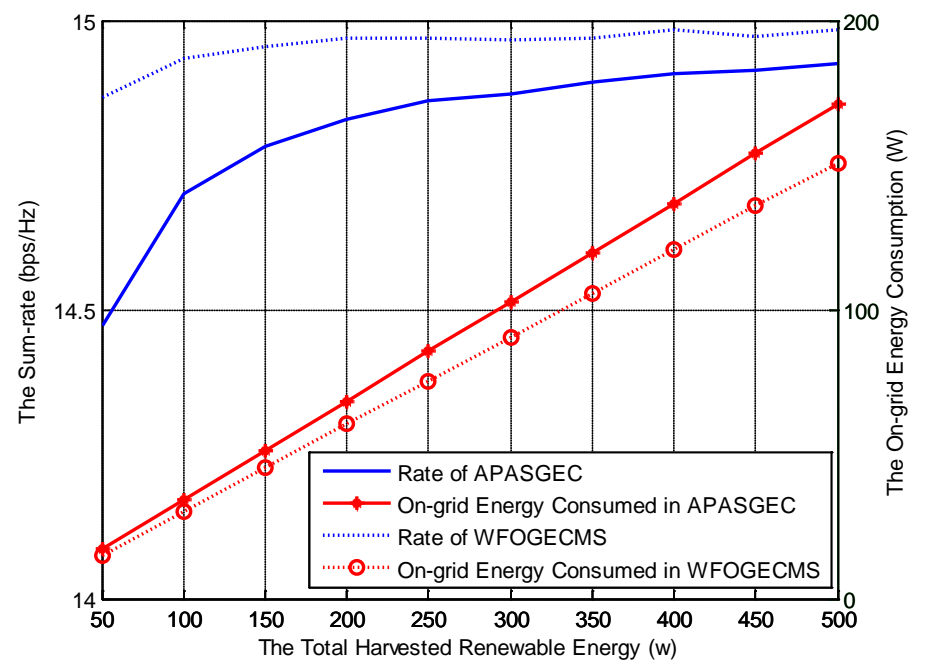

Fig. 6. The sum-rate and grid power consumption VS the total harvest renewable energy

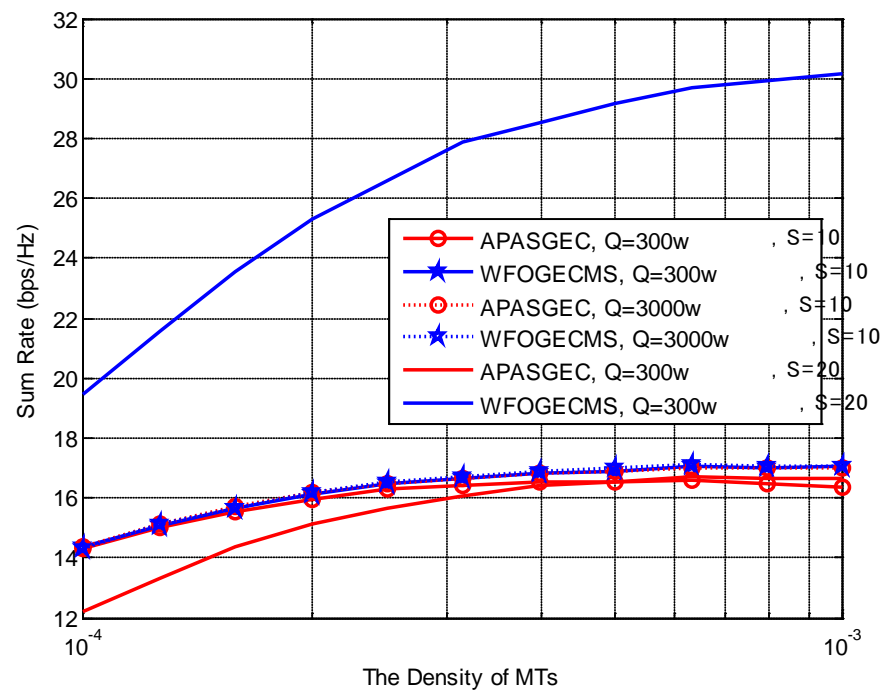

Fig. 7. The sum-rate VS the density of MTs

Finally, In Fig. 7, we show the sum-rate performance VS the density of MTs with $\mathrm{N}=10$ PBs, and $M=30$ MTs. It is observed that, firstly, when $S=10$ base stations, with the density of 
MTs' increases, the sum-rate performance in WFOGECMS and APASGEC almost the same, no matter the total harvest renewable energy $\mathrm{Q}=300$ or 3000 watt. That means, when $\mathrm{Q}$ is big enough, the increase of $\mathrm{Q}$ cannot bring the sum-rate lift. Secondly, when $\mathrm{Q}=300$ watt, the sum-rate performance in the WFOGECMS algorithm with $S=20$ base stations outperforms APASGEC scheme, but almost the same when $S=10$ base stations, no matter what the density of MTs is. That means, the increase of $S$ brings a significant increase in the sum-rate. That mainly because: when $\mathrm{Q}$ is big enough, power is no longer the most important factor, the bandwidth provided by base stations becomes a bottleneck of the sum-rate lift. And the CC adjusts the renewable energy allocation among PBs dynamically and employ green energy cooperation adaptively. So the increase in the number of base stations brings a significant increase in the sum-rate with WFOGECMS algorithm.

\section{Conclusions}

In this paper, we proposed a new algorithm joint power allocation optimization and green energy cooperation for designing cellular networks with hybrid energy supplies. By introducing the CC, complexity calculation can be uploaded, and the system parameters can be adjusted by the CC dynamically. Thus, the redistribution of green energy can be implemented easily. With the newly proposed core controller-assisted energy cooperation mechanism among PBs, we formulate the network rate maximization problem in an uplink system. By applying the water filling optimization arithmetic, we develop an efficient solution to optimize the transmit power allocation with low complexity. Furthermore, we show the rate gains by jointly exploiting WFOPAS algorithm and green energy cooperation in cellular networks. It is revealed that, under a practical energy transfer efficiency value, green energy cooperation is beneficial when the harvested renewable energy among PBs is distributed unevenly. And the proposed water filling optimized green energy cooperation management strategy can achieve about $10 \%$ gains of MT's average rate and $20 \%$ reduction of on-grid energy consumption, the performance of cellular networks with hybrid energy supplies is improved.

\section{References}

[1] A. Fehske, G. Fettweis, J. Malmodin and G. Biczok, "The global footprint of mobile communications: The ecological and economic perspective," in Proc. of IEEE Communications Magazine, vol. 49, no. 8, pp. 55-62, August 2011. Article (CrossRef Link).

[2] S. Lambert et al., "World-wide electricity consumption of communication networks," Optical Express, vol. 20, no. 26, pp. B513-524, Mar. 2012. Article (CrossRef Link).

[3] K. Huang, M. Kountouris and V. O. K. Li, "Renewable Powered Cellular Networks: Energy Field Modeling and Network Coverage," in Proc. of IEEE Transactions on Wireless Communications, vol. 14, no. 8, pp. 4234-4247, Aug. 2015. Article (CrossRef Link).

[4] T. Han and N. Ansari, "On Optimizing Green Energy Utilization for Cellular Networks with Hybrid Energy Supplies," IEEE Transactions on Wireless Communications, vol. 12, no. 8, pp. 3872-3882, August 2013. Article (CrossRef Link).

[5] A. Kwasinski and A. Kwasinski, "Increasing sustainability and resiliency of cellular network infrastructure by harvesting renewable energy," IEEE Communications Magazine, vol. 53, no. 4, pp. 110-116, April 2015. Article (CrossRef Link).

[6] B. Medepally and N. B. Mehta, "Voluntary Energy Harvesting Relays and Selection in Cooperative Wireless Networks," in Proc. of IEEE Transactions on Wireless Communications, vol. 9, no. 11, pp. 3543-3553, November 2010. Article (CrossRef Link). 
[7] V. Raghunathan, S. Ganeriwal and M. Srivastava, "Emerging techniques for long lived wireless sensor networks," IEEE Communications Magazine, vol. 44, no. 4, pp. 108-114, April 2006. Article (CrossRef Link).

[8] Huawei, Mobile networks go green. Article (CrossRef Link).

[9] T. Han and N. Ansari, "Powering mobile networks with green energy," IEEE Wireless Communications, vol. 21, no. 1, pp. 90-96, February 2014. Article (CrossRef Link).

[10] G. Piro et al., "HetNets Powered by Renewable Energy Sources: Sustainable Next-Generation Cellular Networks," IEEE Internet Computing, vol. 17, no. 1, pp. 32-39, Jan.-Feb. 2013. Article (CrossRef Link).

[11] Y. Mao, J. Zhang and K. B. Letaief, "A Lyapunov Optimization Approach for Green Cellular Networks With Hybrid Energy Supplies," IEEE Journal on Selected Areas in Communications, vol. 33, no. 12, pp. 2463-2477, Dec. 2015. Article (CrossRef Link).

[12] K. Huang and V. K. N. Lau, "Enabling Wireless Power Transfer in Cellular Networks: Architecture, Modeling and Deployment," IEEE Transactions on Wireless Communications, vol. 13, no. 2, pp. 902-912, February 2014. Article (CrossRef Link).

[13] L. Liu, R. Zhang and K. C. Chua, "Multi-Antenna Wireless Powered Communication With Energy Beamforming," IEEE Transactions on Communications, vol. 62, no. 12, pp. 4349-4361, Dec. 2014. Article (CrossRef Link).

[14] H. Chen, Y. Li, J. L. Rebelatto, B. F. Uchôa-Filho and B. Vucetic, "Harvest-Then-Cooperate: Wireless-Powered Cooperative Communications," IEEE Transactions on Signal Processing, vol. 63, no. 7, pp. 1700-1711, April1, 2015. Article (CrossRef Link).

[15] H. Tabassum, E. Hossain, M. J. Hossain and D. I. Kim, "On the Spectral Efficiency of Multiuser Scheduling in RF-Powered Uplink Cellular Networks," IEEE Transactions on Wireless Communications, vol. 14, no. 7, pp. 3586-3600, July 2015. Article (CrossRef Link).

[16] W. Ni and X. Dong, "Energy Harvesting Wireless Communications With Energy Cooperation Between Transmitter and Receiver," IEEE Transactions on Communications, vol. 63, no. 4, pp. 1457-1469, April 2015. Article (CrossRef Link).

[17] J. Xu and R. Zhang, "CoMP Meets Smart Grid: A New Communication and Energy Cooperation Paradigm," IEEE Transactions on Vehicular Technology, vol. 64, no. 6, pp. 2476-2488, June 2015. Article (CrossRef Link).

[18] Y. K. Chia, S. Sun and R. Zhang, "Energy Cooperation in Cellular Networks with Renewable Powered Base Stations," IEEE Transactions on Wireless Communications, vol. 13, no. 12, pp. 6996-7010, Dec. 2014. Article (CrossRef Link).

[19] X. Zhang et al., "Macro-assisted data-only carrier for $5 \mathrm{G}$ green cellular systems," IEEE Communications Magazine, vol. 53, no. 5, pp. 223-231, May 2015. Article (CrossRef Link)

[20] X. Zhang, Y. Zhang, R. Yu, W. Wang and M. Guizani, "Enhancing spectral-energy efficiency forLTE-advanced heterogeneous networks: a users social pattern perspective," IEEE Wireless Communications, vol. 21, no. 2, pp. 10-17, April 2014. Article (CrossRef Link)

[21] X. Zhang et al., "Energy-efficient multimedia transmissions through base station cooperation over heterogeneous cellular networks exploiting user behavior," IEEE Wireless Communications, vol. 21, no. 4, pp. 54-61, August 2014. Article (CrossRef Link)

[22] J. Zhang, X. Zhang and W. Wang, "Cache-Enabled Software Defined Heterogeneous Networks for Green and Flexible 5G Networks," IEEE Access, vol. 4, no. , pp. 3591-3604, 2016. Article (CrossRef Link)

[23] J. Xu, L. Duan and R. Zhang, "Cost-aware green cellular networks with energy and communication cooperation," IEEE Communications Magazine, vol. 53, no. 5, pp. 257-263, May 2015. Article (CrossRef Link).

[24] H. Gao, W. Ejaz and M. Jo, "Cooperative Wireless Energy Harvesting and Spectrum Sharing in 5G Networks," IEEE Access, vol. 4, pp. 3647-3658, 2016. Article (CrossRef Link).

[25] J. Zhang, X. Yang, Q. Yao, X. Ge, Minho Jo, and G. Mao, “Cooperative Energy Efficiency Modeling and Performance Analysis in Co-Channel Interference Cellular Networks,” The Computer Journal, Vol. 56, No. 8, pp. 1010-1019, August 2013. Article (CrossRef Link). 
[26] O. Ozel, K. Tutuncuoglu, J. Yang, S. Ulukus and A. Yener, "Transmission with Energy Harvesting Nodes in Fading Wireless Channels: Optimal Policies," IEEE Journal on Selected Areas in Communications, vol. 29, no. 8, pp. 1732-1743, September 2011. Article (CrossRef Link).

[27] X. Kang, Y. K. Chia, C. K. Ho and S. Sun, "Cost Minimization for Fading Channels With Energy Harvesting and Conventional Energy," IEEE Transactions on Wireless Communications, vol. 13, no. 8, pp. 4586-4598, Aug. 2014. Article (CrossRef Link).

[28] T. Han and N. Ansari, "Green-energy Aware and Latency Aware user associations in heterogeneous cellular networks," in Proc. of 2013 IEEE Global Communications Conference (GLOBECOM), Atlanta, GA, pp. 4946-4951, 2013. Article (CrossRef Link).

[29] J. Gong, J. S. Thompson, S. Zhou and Z. Niu, "Base Station Sleeping and Resource Allocation in Renewable Energy Powered Cellular Networks," IEEE Transactions on Communications, vol. 62, no. 11, pp. 3801-3813, Nov. 2014. Article (CrossRef Link).

[30] R. Zhang and C. K. Ho, "MIMO Broadcasting for Simultaneous Wireless Information and Power Transfer," IEEE Transactions on Wireless Communications, vol. 12, no. 5, pp. 1989-2001, May 2013. Article (CrossRef Link).

[31] Boyd, Vandenberghe and Faybusovich, "Convex Optimization," IEEE Transactions on Automatic Control, vol. 51, no. 11, pp. 1859-1859, Nov. 2006. Article (CrossRef Link).

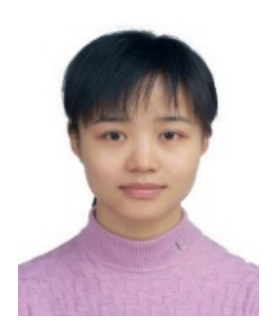

LIN WANG (wanglin121@gmail.com) received the M.E degree in Information and Communication Engineering 2009 from Chongqing University of Posts and Telecommunications, Chongqing, China. She is currently pursuing the Ph.D. degree in the Key Laboratory of Universal Wireless Communications, School of Information and Communication Engineering, Beijing University of Posts and Telecommunications (BUPT), Beijing, China. Her research interests are mainly wireless communications and networks, green communication, cooperative communications and 5G network architecture.

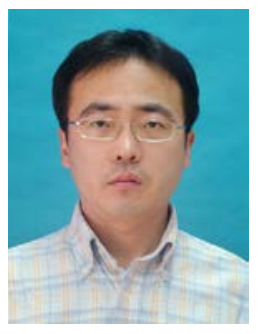

XING ZHANG [M'10, SM'14] (zhangx@ieee.org) is now a full professor with the School of Information and Communications Engineering, Beijing University of Posts and Telecommunications (BUPT), China. His research interests are mainly in 5G wireless communications and networks, green communications, cognitive radio and cooperative communications, big data, and the Internet of Things. He is the author/coauthor of two technical books and more than 60 papers in top journals and international conferences, and has filed more than 30 patents. He has served on the Editorial Boards of several international journals, including IEEE Access, KSII Transactions on Internet and Information Systems and the International Journal of Distributed Sensor Networks, and as a TPC Co-Chair/Symposium Chair/TPC member for a number of major international conferences, including MobiQuitous 2012, IEEE ICC/GLOBECOM/WCNC/ICCC, CR OWNCOM, Chinacom, and others. He received the Best Paper Award at Chinacom 2014 and the 17th International Symposium on Wireless Personal Multimedia Communications, Sydney, Australia.

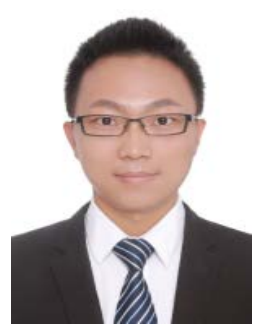

KUN YANG (ykgaoshou@gmail.com) received the B.E and M.E degrees from Shandong Normal University in 2010 and 2013, respectively. He is currently pursuing the Ph.D. degree in the School of Information and Communication Engineering, Beijing University of Posts and Telecommunications (BUPT), China. His research interests are mainly in green communications, $5 \mathrm{G}$ wireless communications and networks, small cell enhancement technologies and cooperative communications 